\title{
The Standard Model of Quantum Measurement Theory: History and Applications
}

\author{
Paul Busch \\ Department of Applied Mathematics \\ The University of Hull \\ Hull, HU6 7RX, England \\ E-mail: p.busch@ maths.hull.ac.uk
}

PEKKa J. LAHTI

Department of Physics

University of Turku

SF-20500 Turku 50, Finland

E-mail: pekka.lahti@utu.fi

\begin{abstract}
The standard model of the quantum theory of measurement is based on an interaction Hamiltonian in which the observable-to-be-measured is multiplied with some observable of a probe system. This simple Ansatz has proved extremely fruitful in the development of the foundations of quantum mechanics. While the ensuing type of models has often been argued to be rather artificial, recent advances in quantum optics have demonstrated their prinicpal and practical feasibility. A brief historical review of the standard model together with an outline of its virtues and limitations are presented as an illustration of the mutual inspiration that has always taken place between foundational and experimental research in quantum physics.
\end{abstract}

\section{Introduction.}

In Chapter Three of his classic The Philosophy of Quantum Mechanics ${ }^{(1)}$, Max Jammer reviews the debate about the interpretation of the indeterminacy relation for position and momentum. He points out 'that of all the interpretations listed ... the following two proved most important for the development of quantum mechanics:

1. The nonstatistical interpretation $\mathrm{I}_{1}$ according to which it is impossible, in principle, to specify precisely the simultaneous values of canonically conjugate variables that describe the behavior of single (individual) physical systems,

2. The statistical interpretation $\mathrm{I}_{2}$ according to which the product of the standard deviations of two canonically conjugate variables has a lower bound given by $h / 4 \pi .^{\prime}$ 
He then proceeds to show that under the measurement-theoretic assumption of the measurements involved being of the first kind, that is, repeatable, $\mathrm{I}_{1}$ is a consequence of $\mathrm{I}_{2}$. The Chapter concludes with the observation 'that certain modern developments have cleared the way for the establishment of theories on the simultaneous measurement of incompatible observables', but it is pointed out that 'little consensus has been reached on the very basic issues of such theories, primarily, it seems, because of diverging definitions of "simultaneous measurements." It is certainly too early to form a balanced judgement on the legitimacy and prospects of such theories.'

Since the time when these lines were written (around 1974) much has happened in theoretical as well as experimental respects. Experimentally, there have been a variety of illustrations of the scatter relation, for example, by means of diffraction experiments involving beams of photons, electrons, or neutrons. On the conceptual side, developments that had started in the mid-1960s have led to a wider concept of observables which allows the formulation of joint observables for collections of noncommuting quantities. Quite independently, measurement-theoretical models were developed which were intuitively interpreted as simultaneous position-momentum measurements. For a long time, both the conceptual innovations and the concrete models remained largely unnoticed, mainly, because their intimate relationship was not made explicit. In fact a new and wider mathematical representation of observables would have to be justified in terms of - at least model - applications; and the models of joint measurements did not allow for any reasonable explanation in terms of the conventional quantum mechanical formalism. Meanwhile the situation has changed completely in that the models have been satisfactorily linked with the new observable concept, and search has begun for possible experimental realisations of the corresponding 'simultaneous', or joint measurements.

It was a certain type of measurement model that played an essential role in these developments: in those models the observable-to-be-measured enters the interaction Hamiltonian as a factor, multiplied by an observable of some probe system. In view of its importance, we propose to name this sort of model the standard model of quantum measurement theory. It is the purpose of this contribution to survey 
the main features of the standard model and to show how it leads, in natural ways, to the representation of observables as positive operator valued measures as well as to realisations of joint measurements of noncommuting observables.

\section{A brief history of the standard model.}

Jammer's observation of $\mathrm{I}_{2}$ implying $\mathrm{I}_{1}$ shows that a theory of simultaneous measurements of canonically conjugate, or more general noncommuting pairs of observables must be based on non-repeatable measurements. We observe that logically this gives room for the consideration of a positive reformulation of the nonstatistical interpretation $\mathrm{I}_{1}$ into

3. The individual interpretation $\mathrm{I}_{3}$ according to which it is possible, in principle, to specify unsharply the simultaneous values of canonically conjugate variables that describe the behavior of single (individual) physical systems.

The twofold task is thus defined to (1) introduce appropriate notions of joint observable and joint measurement for noncommuting quantities in accordance with the probabilistic structure of quantum mechanics, and (2) demonstrate the principal realizability of these notions by formulating concrete joint measurement models. In sections 3 and 4 we shall employ the standard model to demonstrate how these goals can be and indeed have been reached. Beforehand we shall recall some instances in the development of quantum mechanics where this sort of model has played a role. It will be seen that a proper understanding of the models in question could not be obtained on the basis of the conventional quantum formalism but was made possible only after the notion of observables was extended appropriately.

Interestingly, the (to our knowledge) very first mathematically rigorous and physically concrete model of quantum measurement theory leads to a non-repeatable measurement of an unsharp position observable. In fact on the last two pages of his book Mathematische Grundlagen der Quantenmechanik ${ }^{(2)}$, von Neumann for-

mulates a measurement scheme as a coupling between two particles - object and probe, effected by an interaction Hamiltonian of the form $\frac{h}{2 \pi i} q \frac{\partial}{\partial r}$ (in modern notation: $Q \otimes P_{1}$, the product of the object position with the probe momentum). This coupling is shown to establish correlations between the object's position $Q$ and the 
'pointer observable' $Q_{1}$, the position of the probe. The ensuing process is intuitively interpreted as a measurement of the object's position. Nevertheless it is evident that the correlations in question are imperfect and it turns out that the statistics of the measurement are not exactly given by the usual quantum mechanical probability distributions for the object's position. Therefore, the scheme presented by von Neumann as an illustration of his measurement theory rather possesses the potential of demonstrating a limitation of the very quantum axiomatics put forward by himself: as we shall see in the next section, the model is adequately described as a measurement not of an observable in von Neumann's sense but of what is nowadays known as an unsharp position observable.

Instead of drawing this conclusion, the standard model has since been regarded by many authors as a fairly good exemplification of an ideal and repeatable measurement of a single observable. This view is not entirely wrong: we shall see that in the class of standard models there are realisations of repeatable measurements. In other cases there is a nonzero measurement inaccuracy, and the measurement is no longer repeatable. Yet this inaccuracy is determined by the indeterminacy of the pointer observable in the probe's initial state and can therefore be made arbitrarily small by appropriate preparation of the probe. In this spirit Aharonov and Bohm ${ }^{(3)}$ propose a standard model form of a momentum measurement as an unsharp measurement of the kinetic energy of a particle in order to disprove a certain form of energy-time uncertainty relation. In this example it can be shown that in the limit of small inaccuracies a remarkable nondisturbance feature arises: near-eigenstates of the measured observable are transformed into near-eigenstates with the same distribution. ${ }^{(4)}$ It is this property of the standard model which gives it a central status in the investigation of quantum non-demolition measurement schemes. The nondisturbance properties of such measurements almost require them to be of the standard model type. ${ }^{1}$

Rather than concentrating on the small-inaccuracy limit of the standard model, one might consider whether the unsharpness implemented by the preparation of the

\footnotetext{
${ }^{1}$ For a review of the study of quantum non-demolition measurement schemes and their utilisation in weak signal detection, see, e.g. Ref. 5.
} 
probe is of any positive use. Indeed if the probe is in a pure state, the pointer unsharpness is due to the quantum mechanical indeterminacy of the pointer values; and one could expect that this is what is needed in order to devise simultaneous measurements of noncommuting quantities in the spirit of interpretation $\mathrm{I}_{3}$. Intuitively, it is only a small step to consider combining two measurement schemes of the kind introduced by von Neumann, one for a particle's position, another one for its momentum, and to see if the resulting process would serve as a joint measurement. More precisely, one would couple two probes simultaneously with the same particle by introducing an interaction Hamiltonian of the form $\lambda Q \otimes P_{1} \otimes I_{2}+\mu P \otimes I_{1} \otimes P_{2}$ ( $\lambda, \mu$ being coupling constants). This would amount to an extension of the standard model so as to include the measurement of several observables of one object. Such a model was first formulated by Arthurs and Kelly ${ }^{(6)}$ in 1965. It is interesting to note that a variant of the Arthurs-Kelly model was studied independently in DeWitt's Varenna lecture of $1970^{(7)}$, who also makes extensive use of the standard model in illustrating the many-worlds interpretation of quantum mechanics. Still, at that time the term simultaneous measurement could only be used in an informal sense since no formal counterpart to the measurement outcomes and their statistics was known that could have served as a representation of the measured observable in this model. Accordingly, rather than analysing the measurement statistics and drawing the conclusion that they cannot be represented by spectral measures of some self-adjoint operators, these authors, as well as many others afterwards, base their measurement criterion just on the reproduction of expectation values and variances. This procedure has the disadvantage that it does not single out a particular observable as the measured one. We shall see in section 4 that the Arthurs-Kelly measurement scheme does determine a unique phase space observable as the one whose statistics equals the pointer statistics. This opens the way for a rigorous measurement theoretical justification of Heisenberg's individual interpretation $\mathrm{I}_{3}$ of the indeterminacy relation. ${ }^{(8)}$

The possibility of adjusting the measurement inaccuracy within the family of standard models brings about a new approach towards a new understanding of the classical domain within quantum mechanics. For example, the Arthurs-Kelly 
model of a phase space measurement, if appropriately generalized so as to allow for arbitrary pointer preparations, is found to display features typical of a classical measurement situation in the limit of very large position and momentum inaccuracies. $^{2}$ The importance of quantum measurements with large inaccuracies was also recognized by Aharonov et $\mathrm{al}^{(10)}$ when they discovered a 'surprising quantum effect' of classically possible but quantum mechanically nonexistent readings in a standard model treatment of a spin measurement with large pointer variances.

Turning now to the question of the experimental realization of the standard model, it is tempting to refer to the Stern-Gerlach experiment as perhaps the first example that comes close to this type of measurement. However, the usual textbook description, which is based essentially on the interaction Hamiltonian $H=\mu s_{z} \otimes$ $\left(B_{o}+b z\right)$ and the momentum $p_{z}$ as the pointer, can at best be regarded as a caricature of the real situation as the magnetic field is far more complicated than is assumed in the above and indeed ought to satisfy Maxwell's equations. ${ }^{3}$

The question whether the phase space measurement version of the standard model allows for experimental realizations has been answered in the positive only rather recently in the context of quantum optics. Observing that the phase and amplitude quadrature components of a photon field are canonically conjugate quantities, Stenholm ${ }^{(11)}$ showed that the Arthurs-Kelly coupling arises as an approximation to a realistic quantum optical interaction. Another realization that involves only one probe system is obtained by a simple beam splitter coupling between a signal and local mode, followed by a homodyne detection. This model was derived by Leonhardt and Paul ${ }^{(12)}$ as a simplification of a more sophisticated phase measurement arrangement proposed by Noh, Fougeres and Mandel.(13)

\section{Quantum measurement theory and the standard model.}

From the present-day point of view, measurement theory as introduced by von Neumann was restricted to the case of ideal measurements of sharp observables represented by discrete self-adjoint operators. ${ }^{(14)}$ We shall briefly recall this case in order to introduce the ideas of the measurement theory as employed in its standard

\footnotetext{
${ }^{2}$ For details, see Ref. 9.

${ }^{3}$ For an outline of a more realistic account and some relevant references, see Ref. 9.
} 
model.

Let $\mathcal{S}$ be the system on which a measurement is to be performed, and assume that this system is represented by a (complex, separable) Hilbert space $\mathcal{H}$. Assume further that one intends to measure an observable of $\mathcal{S}$ given by a discrete selfadjoint operator $A=\sum a_{i} P_{i}$, with the eigenvalues $a_{i}$ and with the associated eigenprojections $P_{i}, i=1,2, \cdots$. If $\mathcal{S}$ is in a vector state $\varphi$ (a unit vector of $\mathcal{H}$ ), then $p_{\varphi}^{A}\left(a_{i}\right)=\left\langle\varphi \mid P_{i} \varphi\right\rangle$ is the probability that a measurement of $A$ leads to the result $a_{i}$. Consider now another system $\mathcal{A}$, the measuring apparatus or the probe system, associated with the Hilbert space $\mathcal{K}$, and assume that it is prepared independently in a vector state $\phi$, which is taken to be fixed. Let $U: \mathcal{H} \otimes \mathcal{K} \rightarrow \mathcal{H} \otimes \mathcal{K}$ be a unitary operator with the property

$$
U(\varphi \otimes \phi)=\sum P_{i} \varphi \otimes \phi_{i}
$$

where $\left\{\phi_{i}\right\}$ is a fixed set of mutually orthogonal unit vectors in $\mathcal{K}$. Let $Z=\sum z_{i} Z_{i}$ be an observable of $\mathcal{A}$, the pointer observable, such that $Z \phi_{i}=z_{i} \phi_{i}$ for all $i=$ $1,2 \cdots$. Let $\mathcal{R}_{\mathcal{A}}(P[U(\varphi \otimes \phi)])$ be the partial trace of the vector state $U(\varphi \otimes \phi)$ over the object Hilbert space $\mathcal{H}$. We call it the (reduced) state of $\mathcal{A}$ after the measurement. Since $U$ and $\phi$ are assumed to be fixed, we denote this state as $W(\varphi)$. Clearly, $W(\varphi)=\sum p_{\varphi}^{A}\left(a_{i}\right) P\left[\phi_{i}\right]$, and one has for any $i$ and for all $\varphi$,

$$
p_{\varphi}^{A}\left(a_{i}\right)=p_{W(\varphi)}^{Z}\left(z_{i}\right)
$$

showing that the measurement outcome probabilities for $A$ in any initial state $\varphi$ of the object system are recovered as the distribution of the pointer values in the final apparatus state $W(\varphi)$. In this sense the system $\mathcal{A}$, with the Hilbert space $\mathcal{K}$, its initial preparation $\phi$, the pointer observable $Z$, and the measurement coupling $U$ constitute a measurement of $A$. We let $\mathcal{M}=\langle\mathcal{K}, \phi, Z, U\rangle$ denote this measurement. It is obvious that the items in this 4-tuple can be generalised and altered in many ways to produce various kinds of measurements of $A$. The essential point is that any such $\mathcal{M}$ is to fulfill the condition (2) in order serve as a measurement of the observable $A$. The particular measurement scheme sketched above is due to von Neumann and it has a number of special properties. It is a first kind, repeatable, and ideal measurement of the sharp discrete observable $A$. 
There is another reading of condition (2). Given any measurement scheme $\mathcal{M}=\langle\mathcal{K}, \phi, Z, U\rangle$, Equation (2), when stipulated to hold for all $\varphi$ and for all $i$, defines the observable measured by this scheme. We shall go on to apply this point of view to the case of the standard measurement couplings

$$
U=e^{i \lambda A \otimes B}
$$

where $A$ is the observable intended to be measured, $\lambda$ is a coupling constant and $B$ is an observable of $\mathcal{A}$.

Assume still that $A$ is discrete, $A=\sum a_{i} P_{i}$. In this case $U$ may be written in the form $U=\sum P_{i} \otimes e^{i \lambda a_{i} B}$ so that for any $\varphi$ and for (fixed) $\phi$,

$$
U(\varphi \otimes \phi)=\sum P_{i} \varphi \otimes \phi_{\lambda a_{i}}^{B}
$$

where we have introduced the unit vectors $\phi_{\lambda a_{i}}^{B}:=e^{i \lambda a_{i} B} \phi$. Let the pointer observable be denoted by $Z=\sum z_{i} Z_{i}$, where the $z_{i}$ are the distinct eigenvalues, with the mutually orthogonal eigenprojections $Z_{i}$ satisfying $\sum Z_{i}=I$. We shall also use notations like $Z: z_{i} \mapsto Z_{i}$ to indicated the associated positive operator valued (here: spectral) measure. Observe that the vectors $\phi_{\lambda a_{i}}^{B}$ need not be be mutually orthogonal, nor eigenvectors of $Z$. In any case, the condition

$$
p_{\varphi}^{E}\left(a_{i}\right):=p_{W(\varphi)}^{Z}\left(z_{i}\right)
$$

when stipulated to hold for all $\varphi$ and for all $i$, defines the measured observable $E: a_{i} \rightarrow E_{i}$. Note that the relation (5) also induces a pointer function $z_{i} \mapsto a_{i}$, so that a reading $z_{i}$ uniquely indicates a value $a_{i}$ of the observable to be measured. Since the final apparatus state has the form $W(\varphi)=\sum_{i} p_{\varphi}^{A}\left(a_{i}\right) P\left[\phi_{\lambda a_{i}}^{B}\right]$, a direct computation gives

$$
E_{i}=\sum_{j} p_{\phi_{\lambda a_{j}}^{B}}^{Z}\left(z_{i}\right) P_{j}
$$

The operators $E_{i}$ are positive and bounded by the unit operator, $O \leq E_{i} \leq I$, and they sum up to the unit operator, $\sum E_{i}=I$. This is to say that the mapping $E: a_{i} \mapsto E_{i}$ constitutes a (discrete) positive operator valued measure. Furthermore, 
the structure of the operators $E_{i}$ show that they are weigthed means of the spectral projections of $A$ : the measured observable $E$ is a smeared version of the sharp observable $A$. The question at issue is whether one can choose $B, \phi$, and $Z$ such that $E_{i}=P_{i}$, so that the measured observable equals $A$. Before we turn to this question, let us observe that the measurement $\mathcal{M}=\left\langle\mathcal{K}, \phi, Z, e^{i \lambda A \otimes B}\right\rangle$ has the following two properties: the measured observable is commutative, that is,

$$
E_{i} E_{j}=E_{j} E_{i}
$$

for all $i, j=1,2, \cdots$, and the measurement is of the first kind, that is, the probability for a given outcome of the measured observable is the same both before and after the measurement,

$$
p_{\varphi}^{E}\left(a_{i}\right)=p_{T(\varphi)}^{E}\left(a_{i}\right) \text { for all } \varphi \text { and all } i
$$

where $T(\varphi)=\mathcal{R}_{\mathcal{S}}(P[U(\varphi \otimes \phi)])$ is the (reduced) state of $\mathcal{S}$ after the measurement.

We demonstrate next that for $A=\sum a_{i} P_{i}$ one can in general choose $B, \phi$, and $Z$ such that $E_{i}=P_{i}$ for all $i$. To this end we need to ensure that $Z_{i} \phi_{\lambda a_{i}}^{B}=\phi_{\lambda a_{i}}^{B}$ for all $i$, which then entails automatically that $\left\langle\phi_{\lambda a_{i}}^{B} \mid \phi_{\lambda a_{j}}^{B}\right\rangle=0$, for $j \neq i$. We take the probe system to be a particle moving in one-dimensional space, so that $\mathcal{K}=L^{2}(\mathbf{R})$, and couple $A$ with its momentum $P_{1}$ via $U=e^{-i \lambda A \otimes P_{1}}$. Since the momentum generates translations of the position, it is natural to consider choosing the position $Q_{1}$ conjugate to $P_{1}$ as the pointer observable. An initial state $\varphi \otimes \phi$ of the object-probe system is then transformed into $\sum_{i} P_{i} \varphi \otimes \phi_{\lambda a_{i}}^{P_{1}}$. Using the position representation (for $\mathcal{A}$ ) one has $\phi_{\lambda a_{i}}^{P_{1}}(x)=\phi\left(x-\lambda a_{i}\right)$. Assuming that the spacing between the eigenvalues $a_{i}$ of $A$ is greater than $\frac{\delta}{\lambda}$ and that $\phi$ is supported in $\left(-\frac{\delta}{2}, \frac{\delta}{2}\right)$, then the "pointer states" $\phi_{\lambda a_{i}}^{P_{1}}$ are supported in the mutually disjoint sets $\lambda I_{i}$, where $I_{i}=\left(a_{i}-\frac{\delta}{2 \lambda}, a_{i}+\frac{\delta}{2 \lambda}\right)$. This suggests to finally specify the pointer observable $Z: z_{i} \mapsto Z_{i}$ to be a discretised position observable, that is, $z_{i}:=\lambda a_{i}$ and $Z_{i}=E^{Q_{1}}\left(\lambda I_{i}\right)$, the spectral projection of $Q_{1}$ associated with the interval $\lambda I_{i}$. Then Equation (6) gives

$$
E_{i}=\sum_{j} p_{\phi_{\lambda a_{j}}^{Q_{1}}}^{Q_{1}}\left(\lambda I_{i}\right) P_{j}=P_{i}
$$


for each $i$. It follows that the observable measured by this scheme is indeed $A$. Clearly, this measurement is just a realisation of the above abstract measurement scheme of von Neumann.

Let us still consider the coupling (3), but now without assuming that $A$ is discrete. Using the spectral decomposition of $A, A=\int_{\mathbf{R}} a P(d a)$, one may still write $U$ in the form $U=\int_{\mathbf{R}} P(d a) \otimes e^{i a \lambda B}$. An initial state $\varphi \otimes \phi$ of the objectprobe system transforms now

$$
U(\varphi \otimes \phi)=\int_{\mathbf{R}} P(d a) \varphi \otimes \phi_{\lambda a}^{B},
$$

with the notation $\phi_{\lambda a}^{B}:=e^{i a \lambda B} \phi$, and the final apparatus state gets the canonical form

$$
W(\varphi)=\int_{\mathbf{R}} p_{\varphi}^{A}(d a) P\left[\phi_{\lambda a}^{B}\right]
$$

If the pointer observable is a self-adjoint operator $Z$, then the measured observable $E$ is obtained from the condition

$$
p_{\varphi}^{E}(X)=p_{W(\varphi)}^{Z}(\lambda X)
$$

holding for all (Borel) subsets of the real line and for all initial vector states $\varphi$ of $\mathcal{S}$. This gives

$$
E(X)=\int_{\mathbf{R}} p_{\phi_{\lambda a}^{B}}^{Z}(\lambda X) P(d a) .
$$

Clearly, $O \leq E(X) \leq I$, and $E(\mathbf{R})=I$, so that again the mapping $E: X \mapsto E(X)$ is a positive operator valued measure.

The structure of the operators $E(X)$ shows that the actually measured observable $E$ is not the observable $A$, but a smeared version of it. Again, we observe that the measured observable $E$ is commutative, i.e., for all real Borel sets $X, Y$,

$$
E(X) E(Y)=E(Y) E(X)
$$

and the measurement is of the first kind,

$$
p_{\varphi}^{E}(X)=p_{T(\varphi)}^{E}(X), \text { for all } \varphi \text { and for all sets } X,
$$


where $T(\varphi)$ is the final state of $\mathcal{S}$.

The fact that for any $\phi$ and for all $Z$ the measurement $\left\langle\mathcal{K}, \phi, Z, e^{i \lambda A \otimes B}\right\rangle$ is always of the first kind has a remarkable implication: the measured observable $E$ is never $A$ unless $A$ is discrete. Indeed it is known that a first kind measurement of a sharp observable (that is, an observable represented by a self-adjoint operator) is also repeatable and an observable defined by a repeatable measurement is discrete. $^{(14)}$ Thus if $A$ is not discrete the measured observable is always an unsharp version of $A$.

We shall illustrate this important result by taking $A$ to be (a Cartesian component of) the position of $\mathcal{S}, A=Q$. Choosing $B=-P_{1}$ and $Z=Q_{1}$, we get for any $\phi$

$$
E(X)=\int_{\mathbf{R}} p_{\phi_{\lambda q}^{P_{1}}}^{Q_{1}}(\lambda X) E^{Q}(d q)
$$

where $E^{Q}$ denotes the spectral measure of $Q, Q=\int_{\mathbf{R}} q E^{Q}(d q)$. Using the respective spectral representations, the operators $E(X)$ assume the form

$$
E(X)=\iint\left|\phi\left(q^{\prime}-\lambda q\right)\right|^{2} \chi_{\lambda X}\left(q^{\prime}\right) d q^{\prime} E^{Q}(d q)=\chi_{X} * e(Q)
$$

where the function $\left(\chi_{X} * e\right)(y)=\int \chi_{X}(x) e(y-x) d x$ is the convolution of the characteristic function $\chi_{X}$ with the confidence function $e(x):=\lambda|\phi(-\lambda x)|^{2}$. Since $e$ cannot be a delta-function, the measured observable

$$
E: X \mapsto \chi_{X} * e(Q)
$$

is an unsharp position and not the sharp one $E^{Q}: X \mapsto \chi_{X}(Q)$. The standard "position measurement" $\left\langle L^{2}(\mathbf{R}), \phi, Q_{1}, e^{-i \lambda Q \otimes P_{1}}\right\rangle$ introduced by von Neumann thus determines always an unsharp position, where the "unsharpness parameter" $e$ depends on the preparation $\phi$ of the probe and of the coupling constant $\lambda$.

The fact that the measured observable is an unsharp position and not the sharp one can be illustrated in terms of the variance of the measured observable $E$, which is always greater than the variance of $Q$,

$$
\operatorname{Var}(E, \varphi)=\operatorname{Var}(Q, \varphi)+\frac{1}{\lambda^{2}} \operatorname{Var}\left(Q_{1}, \phi\right)
$$


The "noise term" $\frac{1}{\lambda^{2}} \operatorname{Var}\left(Q_{1}, \phi\right)$, which reflects the probe system's quantum nature, can be made small by an appropriate choice of $\phi$, but it can never be eliminated. Only in the limit of strong coupling $\lambda \rightarrow \infty$ would one have $\operatorname{Var}(E, \varphi) \rightarrow \operatorname{Var}(Q, \varphi)$.

\section{A joint position-momentum measurement model.}

In this section we shall provide a model illustration of the important fact that the measurement noise occurring in the von Neumann model and the ensuing unsharp observables may play a fundamental role rather than representing marginal imperfections. To this end we shall combine a standard unsharp position measurement $\left\langle\mathcal{K}_{1}, \phi_{1}, Q_{1}, e^{-i \lambda Q \otimes P_{1}}\right\rangle$ with a standard unsharp momentum measurement $\left\langle\mathcal{K}_{2}, \phi_{2}, P_{2}, e^{i \mu P \otimes Q_{2}}\right\rangle$ to yield a joint position-momentum measurement. Thus we consider a measuring apparatus consisting of two probe systems, $\mathcal{A}=\mathcal{A}_{1}+\mathcal{A}_{2}$, with initial state $\phi_{1} \otimes \phi_{2}$. It will be coupled to the object system $\mathcal{S}$, originally in state $\varphi$, by means of the interaction

$$
U:=\exp \left(-\frac{i}{\hbar} \lambda Q \otimes P_{1} \otimes I_{2}+\frac{i}{\hbar} \mu P \otimes I_{1} \otimes Q_{2}\right)
$$

(In this section we let Planck's constant $\hbar$ explicitly appear in the formulas.) The coupling (20) changes the state of the object-apparatus system $\Psi_{o} \equiv \varphi \otimes \phi_{1} \otimes \phi_{2}$ into $\Psi=U \Psi_{o}$ which in the position representation reads

$$
\Psi\left(q, \xi_{1}, \xi_{2}\right)=\varphi\left(q+\mu \xi_{2}\right) \phi_{1}\left(\xi_{1}-\lambda q-\frac{\lambda \mu}{2} \xi_{2}\right) \phi_{2}\left(\xi_{2}\right)
$$

The measured observable $G$, which is a positive operator valued measure on the (Borel) subsets of $\mathbf{R} \times \mathbf{R}$, is determined from the probability reproducibility conditions

$$
\langle\varphi \mid G(X \times Y) \varphi\rangle:=\left\langle\Psi \mid I \otimes E^{Q_{1}}(\lambda X) \otimes E^{P_{2}}(\mu Y) \Psi\right\rangle
$$

which are to hold for all initial object states $\varphi$ and for all outcome sets $X, Y$. One obtains:

$$
G(X \times Y)=\frac{1}{2 \pi \hbar} \int_{X \times Y} S_{q p} d q d p
$$

The operators $S_{q p}$ are positive and have trace 1 , and they are phase space translates of an operator $S_{0}$ with the same properties. $S_{0}$ depends on the initial probe states 
as well as the coupling constants. As it is rather tedious to write out this connection explicitly, we shall only detail those aspects that are relevant to the present argument.

The question at issue now is what reasons can be given for interpreting the present measurement scheme as a joint measurement of position and momentum. There are four arguments to be put forward here. First, the basic idea formalized above was to apply two measurement procedures simultaneously to the same object system, and this should be expected to yield a joint measurement of the two quantities determined by the separate schemes. Thus we have formulated what one would rightly call a simultaneous measurement.

Second, the observable $G$ defined according to (22), (23) by the simultaneous application of the position and momentum measurement schemes should represent a joint observable for position and momentum. And this is true in the sense that the probability measures given in (22) are proper joint probabilities for a pair of unsharp position and momentum observables. Equivalently, the marginals of the phase space observable (23) are unsharp position and momentum observables:

$$
G(X \times \mathbf{R})=E^{e}(X)=\chi_{X} * e(Q), \quad G(\mathbf{R} \times Y)=F^{f}(Y)=\chi_{Y} * f(P) .
$$

It is straightforward to determine the explicit forms of the confidence functions $e, f$ :

$$
\begin{aligned}
& e(q)=\left\langle q\left|S_{0}\right| q\right\rangle=\int d q^{\prime}\left|\phi_{1}^{(\lambda)}\left(\frac{1}{2} q^{\prime}-q\right)\right|^{2}\left|\phi_{2}^{(\mu)}\left(q^{\prime}\right)\right|^{2}=e_{o} *\left|\phi_{2}^{\left(\frac{\mu}{2}\right)}\right|^{2}(q), \\
& f(p)=\left\langle p\left|S_{0}\right| p\right\rangle=\int d p^{\prime}\left|\hat{\phi}_{2}^{(\mu)}\left(\frac{1}{2} p^{\prime}-p\right)\right|^{2}\left|\hat{\phi}_{1}^{(\lambda)}\left(p^{\prime}\right)\right|^{2}=f_{o} *\left|\hat{\phi}_{1}^{\left(\frac{\lambda}{2}\right)}\right|^{2}(p) .
\end{aligned}
$$

Here we have introduced the scaled functions

$$
\phi_{1}^{(\lambda)}\left(\xi_{1}\right):=\sqrt{\lambda} \phi_{1}\left(\lambda \xi_{1}\right), \quad \phi_{2}^{(\mu)}\left(\xi_{2}\right):=\frac{1}{\sqrt{\mu}} \phi_{1}\left(\frac{1}{\mu} \xi_{2}\right) .
$$

The functions $e_{o}$ and $f_{o}$ are the confidence functions of the original single measurements which can be recovered from the present joint measurement model by switching off one $(\mu=0)$ or the other $(\lambda=0)$ coupling. As indicated by the convolution structure, the original undisturbed inaccuracies are each changed due to the presence of the other device. In other words the simultaneous application of 
the measuring devices for $E^{e_{o}}$ and $F^{f_{o}}$ is not a joint measurement of this original pair of unsharp position and momentum but rather of coarse-grained versions $E^{e}$ and $F^{f}$ of them.

Third, the combination of position and momentum amounts to considering phase space as the value set of an observable. But the phase space is characterized as a homogeneous space of the isochronous Galilei group. Accordingly, a joint observable for position and momentum should also be a phase space observable, that is, it should have the proper covariance under space rotations, space translations and Galilei boosts. In our one-dimensional case we need not take into account the rotation covariance. It turns out that the positive operator valued measure $G$ of Eq. (23) does have the required phase space translation covariance and thus qualifies as a phase space observable.

Finally, one may require that any observable should allow for approximately nondisturbing measurements that are capable of registering the (approximate) values of the observable, without changing that value, whenever the system is in a near-eigenstate. This intuitive idea and postulate can be formalized and it is found that the present scheme does have the corresponding nondisturbance property. ${ }^{(9)}$

The fact that the confidence functions $e, f$ are position and momentum distributions in one and the same "state" $S_{0}$ [Eq. (25)] immediately implies that the measurement unsharpnesses represented by these functions satisfy an uncertainty relation. Nevertheless, it is instructive to see the dynamical mechanism at work in the present model that forces this relation to arise due to the mutual influence of the two measurements being carried out simultaneously. This becomes manifest in the variances of $e$ and $f$ :

$$
\begin{aligned}
& \operatorname{Var}(e)=\frac{1}{\lambda^{2}} \operatorname{Var}\left(Q_{1}, \phi_{1}\right)+\frac{\mu^{2}}{4} \operatorname{Var}\left(Q_{2}, \phi_{2}\right), \\
& \operatorname{Var}(f)=\frac{1}{\mu^{2}} \operatorname{Var}\left(P_{2}, \phi_{2}\right)+\frac{\lambda^{2}}{4} \operatorname{Var}\left(P_{1}, \phi_{1}\right)
\end{aligned}
$$

There are two ways to make the 'undisturbed' variances (the first terms) small: either by choosing large coupling constants or by preparing 'pointer' states having sharply peaked distributions $\left|\phi_{1}\right|^{2},\left|\hat{\phi}_{2}\right|^{2}$. Both options have the same consequence: they produce large contributions to the other quantity's unsharpness (the second 
terms). Thus there is no way of getting both quantities $\operatorname{Var}(e)$ and $\operatorname{Var}(f)$ small in one and the same experiment. Let us evaluate the product of the variances,

$$
\begin{aligned}
& \operatorname{Var}(e) \cdot \operatorname{Var}(f)=\mathcal{Q}+\mathcal{D}, \\
& \mathcal{Q}:=\frac{1}{4} \operatorname{Var}\left(Q_{1}, \phi_{1}\right) \operatorname{Var}\left(P_{1}, \phi_{1}\right)+\frac{1}{4} \operatorname{Var}\left(Q_{2}, \phi_{2}\right) \operatorname{Var}\left(P_{2}, \phi_{2}\right), \\
& \mathcal{D}:=\frac{1}{\lambda^{2} \mu^{2}} \operatorname{Var}\left(Q_{1}, \phi_{1}\right) \operatorname{Var}\left(P_{2}, \phi_{2}\right)+\frac{\lambda^{2} \mu^{2}}{16} \operatorname{Var}\left(Q_{2}, \phi_{2}\right) \operatorname{Var}\left(P_{1}, \phi_{1}\right) .
\end{aligned}
$$

Making use of the uncertainty relations $\operatorname{Var}\left(Q_{k}, \phi_{k}\right) \operatorname{Var}\left(P_{k}, \phi_{k}\right) \geq \hbar^{2} / 4$ for the two probe systems, we find that both terms $\mathcal{Q}, \mathcal{D}$ can be estimated from below. Putting $x:=16 \operatorname{Var}\left(Q_{1}, \phi_{1}\right) \operatorname{Var}\left(P_{2}, \phi_{2}\right) /(\lambda \mu \hbar)^{2}$, we obtain:

$$
\begin{aligned}
& \mathcal{Q} \geq \frac{1}{4}\left(\frac{\hbar^{2}}{4}+\frac{\hbar^{2}}{4}\right)=\frac{\hbar^{2}}{8}, \\
& \mathcal{D} \geq \frac{\hbar^{2}}{16}\left(x+\frac{1}{x}\right) \geq \frac{\hbar^{2}}{8} .
\end{aligned}
$$

This shows finally that

$$
\operatorname{Var}(e) \cdot \operatorname{Var}(f)=\mathcal{Q}+\mathcal{D} \geq \frac{\hbar^{2}}{8}+\frac{\hbar^{2}}{8}=\frac{\hbar^{2}}{4} .
$$

It is remarkable that either one of the terms $\mathcal{Q}$ and $\mathcal{D}$ suffices to provide an absolute lower bound for the uncertainty product. Hence there are two sources of inaccuracy that give rise to an uncertainty relation. Neglecting $\mathcal{D}$, it would be simply the uncertainty relations for the two parts of the apparatus which forbids making the term $\mathcal{Q}$ arbitrarily small. This is in the spirit of Bohr's argument according to which it is the quantum nature of part of the measuring device that makes it impossible to escape the uncertainty relation. Note that the two terms occurring in $\mathcal{Q}$ each refer to one of the probe systems, and they contribute independently to the lower bound for $\mathcal{Q}$; furthermore no coupling parameters appear in $\mathcal{Q}$. There is no trace of a mutual influence between the two measurements being carried out simultaneously. On the other hand neglecting the term $\mathcal{Q}$, one would still be left with the two contributions collected in $\mathcal{D}$, the combination of which has again a lower bound. The terms in $\mathcal{D}$ are products of variances and coupling terms associated with the two probe systems, showing that $\mathcal{D}$ reflects the mutual disturbance of the two measurements. This is in accord with Heisenberg's illustrations of the uncertainty 
relation. For example if a particle is measured so as to have a rather well-defined momentum, then a subsequent measurement of position by means of a slit influences the effect of the preceding momentum measurement to the extent required by the uncertainty relation.

Finally we should like to emphasise that the nature of the measurement 'inaccuracy', or unsharpness, is determined by the preparations of the apparatus. Insofar as the pointer observables are indeterminate and not merely subjectively unknown, this interpretation applies to the measurement uncertainties as well: each individual measurement outcome is intrinsically unsharp, reflecting thereby a genuine quantum noise inherent in the measurement process, so that the inequality (30) should be properly called an indeterminacy relation.

One would expect that being able to perform phase space measurements it should also be possible to observe trajectories of microscopic particles. Thus one may hope to achieve a detailed quantum mechanical account of the formation of cloud or bubble chamber tracks. We shall indicate here that within the present model necessary conditions for such quasi-classical measurement behaviour are "macroscopically" large inaccuracies contributed by the device and, relative to the scale of these inaccuracies, good localisation of the object.

A (quasi-)classical measurement situation is characterised among others by the possibility of observing a particle without necessarily influencing it. The above phase space measurement model allows one to formalise this and some further classicality conditions and to demonstrate their realizability. We shall formulate four such requirements.

First, it should be admissible to think of the particle having "arbitrarily sharp" values of position and momentum. This cannot be meant in an absolute sense but only relative to the scale defined by the resolution of the means of measurement. (C1) Near value determinateness.

$$
\operatorname{Var}(Q, \varphi) \ll \operatorname{Var}(e), \quad \operatorname{Var}(P, \varphi) \ll \operatorname{Var}(f)
$$

Such states may be viewed as "localised" in phase space.

Next, the position and momentum measurements should not disturb each other 
when performed jointly. This can be controlled by the variances (27) in terms of the condition that the additional noise terms should remain negligible:

(C2) Small mutual disturbance. $\operatorname{Var}(e) \simeq \operatorname{Var}\left(e_{o}\right)$ and $\operatorname{Var}(f) \simeq \operatorname{Var}\left(f_{o}\right)$; therefore

$$
\begin{aligned}
\frac{1}{\lambda^{2}} \operatorname{Var}\left(Q_{1}, \phi_{1}\right) & \gg \frac{\mu^{2}}{4} \operatorname{Var}\left(Q_{2}, \phi_{2}\right), \\
\frac{1}{\mu^{2}} \operatorname{Var}\left(P_{2}, \phi_{2}\right) & \gg \frac{\lambda^{2}}{4} \operatorname{Var}\left(P_{1}, \phi_{1}\right) .
\end{aligned}
$$

Third, in view of the uncertainty relation (2.18) for the measurement inaccuracies it should be kept in mind that in a classical measurement the imprecisions seem to be so large that no indication of Planck's constant can ever be observed. (C3) No limit of accuracy. The position and momentum measurement inaccuracies can be made arbitrarily small:

$$
\operatorname{Var}(e) \operatorname{Var}(f) \gg \frac{\hbar^{2}}{4} .
$$

Finally, since the properties to be measured are practically determinate in localized states, one should expect that a measurement will not necessarily disturb the system but will merely register the corresponding values. This is to say that the measurement should be approximately nondisturbing.

(C4) Approximate nondisturbance. Localised states [cf. (C1)] should not be disturbed much in a joint position-momentum measurement.

These features are not mutually independent. It is evident that (C1) implies (C3). The second property, (C2), gives somewhat more:

$$
\operatorname{Var}(e) \operatorname{Var}(f) \geq \operatorname{Var}\left(e_{o}\right) \operatorname{Var}\left(f_{o}\right) \gg \frac{\hbar^{2}}{4}
$$

This shows that mutual nondisturbance can only be achieved if from the outset one starts with highly unsharp measurements. If the requirements expressed in (C1) and (C2) are somewhat strengthened, one can prove by means of the present model that the approximating conditions $(\mathrm{C} 1)$ and $(\mathrm{C} 2)$ are self-consistent, can be realised, and do indeed lead to $(\mathrm{C} 4) .{ }^{(9)}$

In the classical measurement situation described here, one is facing two kinds of uncertainty which have to be interpreted quite differently. If one starts with a 
localised though otherwise unknown state, then it is a matter of subjective ignorance what the "true values" $\left(q_{o}, p_{o}\right)=\left(\langle Q\rangle_{\varphi},\langle P\rangle_{\varphi}\right)$ of position and momentum are. The measurement will give some (point-like) outcome $(q, p)$ most likely in a region around $\left(q_{o}, p_{o}\right)$, for which the probabilities are non-negligible provided that $\left|q-q_{o}\right| \leq$ $n \sqrt{\operatorname{Var}(e)},\left|p-p_{o}\right| \leq n \sqrt{\operatorname{Var}(f)}$, with $n$ of the order of unity. Which result will come out is objectively undecided as the unsharpnesses originate from the pointer indeterminacies. Hence with respect to the state inference problem one is dealing with subjective uncertainties, while predictions of future measurement outcomes are objectively indeterminate.

These considerations reveal the decisive role of Planck's constant $\hbar$ for the classical limit of quantum mechanics in a new sense. Only with respect to measuring instruments yielding macroscopic inaccuracies, Eq. (34), is it possible to neglect the quantum mechanical restrictions and to make approximate use of the classical physical language as laid down in (C1-4). It is remarkable that the indeterminacy product $\operatorname{Var}(Q, \varphi) \operatorname{Var}(P, \varphi)$ of the object and the products $\operatorname{Var}\left(Q_{k}, \phi_{k}\right) \operatorname{Var}\left(P_{k}, \phi_{k}\right)$ of the probe systems need not at all be small for quasi-classical measurements. Thus one can conceive of measuring classical trajectories for microscopic particles.

A more detailed analysis of the state changes incurred by the object system shows that the present model offers a continuous transition between the two extremes of nearly repeatable and nearly nondisturbing (first kind) measurements; thus it becomes evident that these two ideals fall apart into mutually exclusive options for unsharp observables, while in the case of sharp observables the two concepts coincide.

\section{Conclusion: some reflections.}

In the presentation of the standard model we have freely used the two possible alternative readings of the probability reproducibility condition (5): on one hand, this relation serves as a criterion for a measurement scheme to be a measurement of a given observable; on the other hand, the observable actually measured by the scheme is uniquely determined by (5). Thus, starting with a quantum mechanical modelling of a measurement as a physical process, one is inevitably led to introduce 
the measured observable as a positive operator valued measure. From this point of view, it is obvious that quite some experimental ingeniuity is required for realising a measurement of a sharp observable.

It is remarkable that for a long time the quantum measurement theory and the theory of positive operator valued measures developed quite independently and largely without taking notice of each other. That situation may be characterized by saying that the modelling of measurements would be doomed to be blind unless it was carried out in the light of the concept of observables as positive operator valued measures; and that the theory of general observables would remain void of physical content unless it became supplemented with concrete measurement models. Fortunately the two have since been brought together into a fruitful interaction.

There are several possible reasons why the standard model did not by itself give rise to the generalization of the notion of observable required for an understanding of what was being measured. For example, von Neumann came close to the conclusion in question when he calculated the joint probability for the pointer and the observable to be measured; but he only used this result to indicate that the correlation between these quantities could be made arbitrarily, though not absolutely, strong. This shows that he identified the term measurement with repeatable measurement, since he concluded that due to the rather good correlations, his model could be regarded as an approximate realization of a measurement of position. In this view the intrinsic inaccuracy of the measurement is regarded as an unwanted imperfection and not seen as the key to a proper notion of a joint unsharp measurement. Accordingly, von Neumann sketches a concept of joint measurement for position $Q$ and momentum $P$ where two commuting sharp observables $Q^{\prime}, P^{\prime}$ are constructed that are close to $Q$ and $P$, respectively, in some suitable topology (Ref. 2, Section V.4). He regards this procedure as 'purely mathematical'. By contrast, the notion of phase space measurement reviewed in Section 4 is physically quite appealing and not far from experimental realizability.

Another reason why the standard model played no role in introducing the general concept of observables may have been the following. In most of the papers dealing with variants of the standard model, the Heisenberg picture was used instead 
of the Schrödinger picture, which then gave rise only to the conclusion that the pointer observable has the same expectation value as the observable intended to be measured. In addition the proportionality between the Heisenberg operators representing the pointer observable and the observable intended to be measured seemed to suggest taking the latter for the measured observable. However, as we pointed out above, it is (only) the probability reproducibility condition (5) that gives a thorough characterization of the observable actually measured; and this differs, in general, from the intended observable. To be fair, it was recognized that the measurement schemes in question provided only an approximate measurement of the object observable under consideration. Equation (6) illustrates the precise relation between the measured observable and the underlying sharp observable: the former is a smeared version of the latter.

At this point it might appear as if positive operator valued measures, as they emerge in standard model-type measurements, are not much more than a convenient description of imperfect, or inaccurate measurements of "ordinary", sharp observables. It is true that this is one purpose of certain unsharp observables. But it must be noted that not all unsharp observables are commutative, and when they are not, there are no underlying sharp observables of which they were smeared versions. The phase space observable of Section 4 is an example of a kind of experimental question that does not allow for a theoretical formulation on the basis of sharp observables only. In this example it is crucial to realize that there is a genuinely quantum mechanical source of unsharpness, namely, the indeterminacy of the pointer observables in the initial probe states. According to equations (19) and (28), this can be interpreted in accordance with $\mathrm{I}_{3}$ as quantum noise inherent in the measurement outcomes so that the measurement uncertainties satisfy the Heisenberg indeterminacy relation.

The unsharpness inherent in observables that are not represented as self-adjoint operators brings about yet another important innovation into quantum physics. The von Neumann model was found to be an unsharp, non-repeatable measurement of position (Section 3). Considering that the lack of repeatability is related to the fact that the measured observable is continuous, one might try to restore repeatability in 
this model by discretising the pointer observable, as this would amount to defining a discrete, though still unsharp, position $X_{i} \mapsto E\left(X_{i}\right)=\chi_{X_{i}} * e(Q)$. It turns out that the measurement, while always of the first kind, still will not be repeatable. The fact that for measurements of unsharp observables the first kind-property is essentially weaker than the repeatability property comes as an advantage in some respects. Indeed repeatable measurements will produce strong "disturbances" in that they turn non-eigenstates into eigenstates of the measured observable. By contrast, the first kind-property is a "nondisturbance" feature of a measurement as it only ensures that the states are changed so gently that the distribution of the values of the measured observable does not change. It follows that one gains more control over the magnitude of state changes for a larger class of states than just eigenstates (if there are any). This nondisturbance property is what one would expect to be present in a classical measurement situation (Section 4): macroscopic, classical observables have fairly (though perhaps not absolutely) well-defined values which can be detected without being changed.

To conclude, we observe that the assessment of the role of the measurement unsharpness in the standard model has shifted from "marginal", "negligible" or perhaps "undesirable" towards "interesting" or even "crucial" in some circumstances; and this shift went along with the conception of joint measurements of noncommuting quantities and of measurements that are less invasive - and yet sometimes more informative - than sharp measurements.

\section{References.}

1. M. Jammer. The Philosophy of Quantum Mechanics (Wiley, New York, 1974).

2. J. von Neumann. Mathematische Grundlagen der Quantenmechanik (Springer, Berlin, 1932);

English transl.: Mathematical Foundations of Quantum Mechanics (Princeton University Press, Princeton, 1955).

3. Y. Aharonov, D. Bohm, Phys. Rev. 122, 1649 (1961).

4. P. Busch, Found. Phys. 20, 33 (1990).

5. C.M. Caves, K.S. Thorne, R.W.P. Drever, V.P. Sandberg, M. Zimmerman, Rev. Mod. Phys. 52, 341 (1980). D.F. Walls, in Symposium on the Foundations of Modern Physics 1993, eds.

P. Busch, P. Lahti, P. Mittelstaedt (World Scientific, Singapore, 1993).

6. $\quad$ E. Arthurs, J.L. Kelly, Bell Syst. Tech. J. 44, 725 (1965).

7. B.S. DeWitt, in Foundations of Quantum Mechanics, ed. B. d'Espagnat (Academic Press, 
New York, 1972).

8. P. Busch, Doctoral Dissertation, Cologne 1982; English transl.: Int. J. Theor. Phys. 24, 63 (1985). P. Busch, P.J. Lahti, Philosophy of Science 52, 64 (1985).

9. P. Busch, M. Grabowski, P. Lahti, Operational Quantum Physics (Springer-Verlag, Berlin, 1995).

10. Y. Aharonov, D.Z. Albert, L. Vaidman, Phys. Lett. 124A, 199 (1987); Phys. Rev. Lett. 60, 1351 (1988).

11. S. Stenholm, Ann. Phys. (N.Y.) 218, 233 (1992).

12. U. Leonhardt, H. Paul, Phys. Rev. A47, R2460 (1993); U. Leonhardt, Phys. Rev. A48, 3265 (1993).

13. J.W. Noh, A. Fougeres, L. Mandel, Phys. Rev. A45, 424 (1992); Phys. Rev. A46, 2840-2852 (1992).

14. P. Busch, P. Lahti, P. Mittelstaedt, The Quantum Theory of Measurement (Springer-Verlag, Berlin, 1991; 2nd edition in preparation).

\section{Footnotes.}

1. For a review of the study of quantum non-demolition measurement schemes and their utilisation in weak signal detection, see, e.g. Ref. 5.

2. For details, see Ref. 9.

3. For an outline of a more realistic account and some relevant references, see Ref. 9. 